\title{
Therapeutic target for external beam $x$-irradiation in experimental spinal cord injury
}

\author{
Richard J. Zeman, PhD, ${ }^{1}$ Xialing Wen, MS, ${ }^{1}$ Chitti R. Moorthy, MD, ${ }^{2}$ and Joseph D. Etlinger, PhD'1 \\ Departments of ${ }^{1}$ Cell Biology and Anatomy and ${ }^{2}$ Radiation Medicine, New York Medical College, Valhalla, New York
}

OBJECTIVE X-irradiation has been shown to be beneficial to recovery from spinal cord injury (SCI); however, the optimal therapeutic target has not been defined. Experiments were designed to determine the optimal target volume within the injured spinal cord for improving functional recovery and sparing tissue with stereotactic x-irradiation.

METHODS SCI was produced in rats at the T10 level. A 20-Gy dose of radiation was delivered with a single, 4-mmdiameter, circular radiation beam centered either on the injury epicenter or 4 or $8 \mathrm{~mm}$ caudal or rostral to the injury epicenter. Locomotor function was determined for 6 weeks with the Basso, Beattie, and Bresnahan locomotor scale and tissue sparing by histological analysis of transverse sections along the spinal cords.

RESULTS X-irradiation of spinal cord segments at $4 \mathrm{~mm}$, but not $8 \mathrm{~mm}$, caudal or rostral to the contusion epicenter resulted in increases in locomotor recovery. Consistently, significant tissue sparing also occurred with x-irradiation centered at those sites, although irradiation centered $4 \mathrm{~mm}$ rostral to the epicenter led to tissue sparing along the greatest length of the spinal cord. Interestingly, regression analysis of these variables demonstrated that the quantitative relationship between the amount of tissue spared and the improvement in locomotion recovery was greatest in a region several millimeters rostral to the injury epicenter.

CONCLUSIONS These results indicate that $x$-irradiation in a region rostral to the injury epicenter is optimal for recovery from SCl. This minimal target should be attractive for therapeutic application since it allows a greatly reduced target volume so that uninjured tissue is not needlessly irradiated.

https://thejns.org/doi/abs/10.3171/2019.11.SPINE19305

KEYWORDS spinal cord injury; contusion; locomotor function; cavitation; stereotactic x-irradiation

$\mathrm{S}$ TEREOTACTIC radiosurgery is presently used for a variety of clinical applications in areas such as oncology and neurosurgery, since it has an advantage over conventional methods in that specifically defined tissue targets can be noninvasively treated while avoiding damage to surrounding tissue. Recently, another potential application was revealed when, in an experimental model of contusion injury, stereotactic x-irradiation of several segments of an injured spinal cord with a relatively low dose-i.e., $10 \mathrm{~Gy}$-was shown to exert a neuroprotective effect while enhancing the recovery of locomotor function and sparing cord tissue. ${ }^{32}$

Stereotactic X-irradiation is a potentially superior therapeutic modality for spinal cord injury (SCI), with several advantages compared to current steroid treatment with methylprednisolone. X-rays can completely penetrate in- jured spinal cord tissue without requiring compromised circulation for delivery of the therapeutic agent, as in the case of systemically administered methylprednisolone, thereby avoiding undesired side effects, such as myopathy and immunosuppression, in noncord tissues. ${ }^{23}$ Targeted $\mathrm{x}$ irradiation may also be beneficial both by expanding the volume of injured tissue that can be treated and the duration of the therapeutic time window, ${ }^{30}$ each of which can be decreased by compromised circulation due to injury.

In the experiments in which stereotactic x-irradiation of injured spinal cord tissue enhanced locomotor recovery and spared cord tissue after contusion injury, ${ }^{32}$ the injured spinal cord was identified as the therapeutic target of irradiation, rather than surrounding noncord tissue. However, the precise anatomical location of cord tissue necessary to show a benefit from radiosurgery in relation to the site of 
the contusion injury is not known. This is in contrast to treatment of other tissue targets, such as tumors, in which the exact dimensions can be readily detected with scanning technology and then targeted for irradiation. The location of the irradiated portion of spinal cord that was associated with improved recovery was centered on and included the injury epicenter, as well as contiguous, uninjured regions rostral and caudal to the injury site, and it is possible that a more optimal target is located at some point within the irradiated region. For example, providing neuroprotection for corticospinal or propriospinal axonal tracts $^{7}$ originating rostral to the injury may be most critical for promoting recovery. Alternatively, treatment of the injury site itself, where tissue loss is greatest, or caudally, where axonal sprouting supporting locomotor recovery occurs, ${ }^{1}$ could be more beneficial. Additionally, there appear to be postinjury rostral-caudal gradients of tissue damage and recovery that, in turn, may differentially determine the relative effectiveness of localized treatment. ${ }^{20,22,27}$ To determine the location of the optimal therapeutic target, experiments were designed to $\mathrm{x}$-irradiate portions of the spinal cord located exclusively caudal to, rostral to, or at the injury epicenter.

\section{Methods}

Adult female (220-260 g) Wistar rats were acquired from Charles River Breeding Laboratories and were quartered in a temperature-regulated $\left(23^{\circ} \mathrm{C}\right)$ animal facility. As in prior studies, ${ }^{2,3,13,28-31}$ a weight-drop apparatus similar to the NYU impactor ${ }^{11}$ was used to contuse the spinal cords at the level of T10. The rats were anesthetized before surgery with an intraperitoneal injection of pentobarbital sodium $(60 \mathrm{mg} / \mathrm{kg})$. A T9-10 laminectomy was then performed aseptically to expose the spinal cord. To prevent movement during the contusion injury, the spinous processes at $\mathrm{T} 8$ and T11 were immobilized with clamps. In order to maintain body temperature at $37^{\circ} \mathrm{C}$ during the period of surgery, a temperature-regulated heating pad and rectal thermometer were used. Groups of rats were contused by dropping a $10-\mathrm{g}$ rod with a tip diameter of $2.5 \mathrm{~mm}$ from an elevation of $25 \mathrm{~mm}$ onto the exposed spinal cord dura mater. The spinal cords were exposed dorsally to a 20-Gy dose of x-irradiation at 20 minutes postinjury. We used a GE Maximar (C.P. Type 1, General Electric) x-irradiator with a single, 4-mm-diameter, circular radiation beam centered either on the injury epicenter or 4 or $8 \mathrm{~mm}$ caudal or rostral to the injury epicenter (Fig. 1A). Prior to irradiation, additional laminectomy to $10 \mathrm{~mm}$ rostral or caudal to the epicenter was performed to expose the spinal cord to irradiation. Control rats received dorsal irradiation of the thorax, rather than the spinal cord, with the beam centered $1 \mathrm{~cm}$ lateral to the SCI epicenter or were untreated. In all, there were 7 treatment groups with 12 rats $\mathrm{x}$-irradiated at the injury epicenter, 13 rats $x$-irradiated $4 \mathrm{~mm}$ caudally, 13 rats $x$-irradiated $8 \mathrm{~mm}$ caudally, 12 rats x-irradiated 4 $\mathrm{mm}$ rostrally, 12 rats $\mathrm{x}$-irradiated $8 \mathrm{~mm}$ rostrally, 13 rats $\mathrm{x}$-irradiated $10 \mathrm{~mm}$ laterally, and 11 rats not irradiated. Each incision was closed after contusion with wound clips, and the rats received $0.02 \%$ amoxicillin in their drinking water for the remainder of the experiment to prevent infec- tion. For 2-3 weeks, the bladders were expressed manually twice daily until automaticity returned. All of the procedures involving vertebrate animals were approved by New York Medical College's Institutional Animal Care and Use Committee and were in compliance with the Guide for the Care and Use of Laboratory Animals.

\section{Behavioral Analysis}

The degree of recovery of locomotor function was evaluated using the Basso, Beattie, and Bresnahan (BBB) locomotor scale, ${ }^{2-4}$ which has scores ranging from 0 (total paralysis) to 21 (near-normal locomotion). Scores of 1-8 indicate small or large movements of the 3 joints of the hindlimb, a score of 9 signifies weight-bearing status or dorsal stepping, and scores of 10-20 indicate progressive advancements in coordinated walking ability. The rats were acclimated daily to a circular examination area 3 feet in diameter for a week prior to surgery. For 3 consecutive days following contusion and at 1-week intervals thereafter up to 6 weeks postinjury, each rat was scored for locomotor function according to the BBB scale. The locomotor scores for each animal were determined by two observers blinded to the treatment conditions during each 4-minute observation period of open field testing, and the scores for each of the hindlimbs were averaged to obtain the mean score for each session.

\section{Spinal Cord Histomorphometry}

The spinal cords were fixed by transcardial perfusion in the anesthetized rats (pentobarbital sodium, $60 \mathrm{mg} / \mathrm{kg}$, intraperitoneal injection) with phosphate-buffered saline (pH 7.4) containing $1 \%$ glutaraldehyde and $4 \%$ paraformaldehyde, directly following the final behavioral evaluation session. The cords were then dissected and embedded in paraffin for serial sectioning with a microtome. The spinal cords were cross-sectioned at a thickness of $15 \mu \mathrm{m}$ from $\mathrm{T} 8$ to $\mathrm{T} 12$, which included the contusion site, that could be visualized externally. The sections were stained for myelin with Luxol fast blue and counterstained with cresyl violet, as in previous studies. ${ }^{3}$ The cross-sectional areas of spared spinal cord white matter were measured with planimetry software (SigmaScan, Systat Software Inc.) from digitized images (Photometrics) of the stained section exhibiting the least remaining areas of white matter, i.e., the injury epicenter, from each spinal cord without the measurement readers' knowledge of the treatment conditions. Cross-sectional areas of spared white matter were then measured at 500-um intervals along the spinal cord extending $4-5 \mathrm{~mm}$ rostrally and caudally from the contusion epicenter.

\section{Statistical Analysis}

Evaluation of the statistical significance of the effects of x-irradiation treatment on locomotor scores was determined by mixed factorial analysis of variance with repeated measures and by Duncan's multiple-range post hoc test (SPSS version 13.0). Between-group comparisons of differences in the cross-sectional areas of spinal cord white matter were performed using the least significant difference multiple range test (SPSS version 13.0). Regres- 

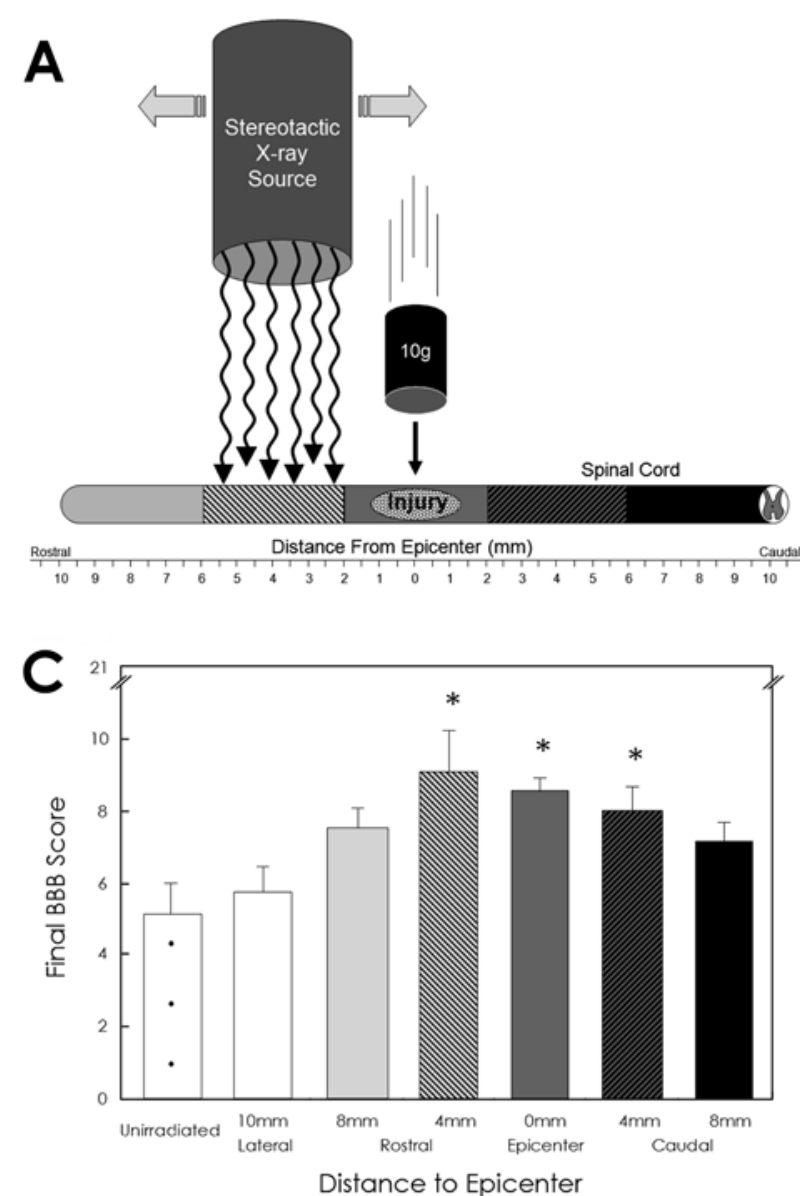

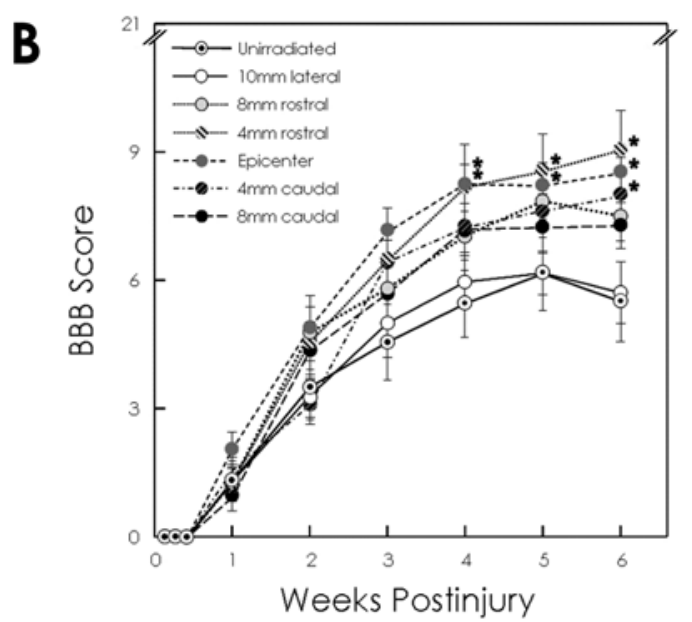

FIG. 1. A: Diagram of the experimental design. Groups of rats received contusion injury with a $10-\mathrm{g}$ weight dropped $25 \mathrm{~mm}$ onto the exposed spinal cord at T10, and at 20 minutes postinjury, a 20-Gy dose of x-irradiation was delivered dorsally with a single, 4-mm-diameter, circular radiation beam centered either on the injury epicenter or $4 \mathrm{~mm}$ or $8 \mathrm{~mm}$ caudal or rostral to the injury epicenter. Control rats received dorsal irradiation of the thorax with the beam centered $1 \mathrm{~cm}$ lateral to the SCl epicenter or were not irradiated. B: The effects of varying the rostral-caudal position of the $x$-irradiation beam relative to the contusion site on the time course of recovery of locomotor function. Values are means \pm SE of determinations of locomotor recovery according to the BBB locomotor scale. ${ }^{*} p<0.05$; significant effect of $x$-irradiation on BBB score compared to rats receiving irradiation of the thorax (mixed factorial analysis of variance with repeated measures [group $F\{6,79\}=2.6 ; p=0.024$ ]; [session $F\{5,395\}=204.3 ; p=$ 0.0005]; [group x session interaction $F\{30,395\}=1.4 ; p=0.088$ ]; and Duncan's test post hoc). C: Final BBB scores as in Fig. $1 B$.

sion analysis was performed with SPSS version 13.0. A p value $<0.05$ was considered to be statistically significant.

\section{Results}

Contusion injury impaired locomotor function, as measured by BBB locomotor scores, to a level of complete paralysis for several days following injury (Fig. 1B). Locomotor function then progressively improved within 1-4 weeks of injury to levels that were maintained for the remainder of the 6-week observation period. Similarly, $\mathrm{x}$-irradiation of the thorax at 20 minutes postinjury with a 4-mm-diameter beam centered at $10 \mathrm{~mm}$ lateral to the contusion epicenter led to recovery of locomotor function that was not significantly different than that of unirradiated rats receiving an identical contusion injury. In contrast, $\mathrm{x}$-irradiation of the spinal cord with the beam centered at the contusion epicenter or at either $4 \mathrm{~mm}$, but not $8 \mathrm{~mm}$, caudal or rostral to the contusion epicenter resulted in significantly greater increases in locomotor recovery compared to unirradiated rats or in rats $\mathrm{x}$-irradiated on the thorax (Fig. 1B and C).

Contusion injury causes central cavitation of the spinal cord, with loss mostly of gray matter and sparing of a peripheral rim of white matter (Fig. 2A). Because the extent of axon sparing in the white matter is thought to determine recovery of locomotor function, ${ }^{2,3,21,28}$ the cross-sectional area of spared white matter was determined in myelinstained transverse sections at $0.5-\mathrm{mm}$ intervals along the length of the x-irradiated spinal cords. In parallel with the results of locomotor scoring, significant increases in white matter sparing were also observed only after $\mathrm{x}$-irradiation centered at the epicenter and at $4 \mathrm{~mm}$, but not $8 \mathrm{~mm}$, rostral and caudal to the epicenter in a region extending approximately from $2 \mathrm{~mm}$ rostral to $1.5 \mathrm{~mm}$ caudal to the epicenter (Fig. 2B and C). In addition, the greatest extent of sparing along the length of the cord occurred when x-irradiation was centered $4 \mathrm{~mm}$ rostral to the injury epicenter. 
A
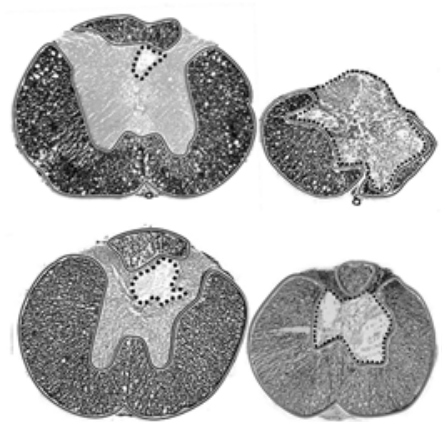

$+3.5$

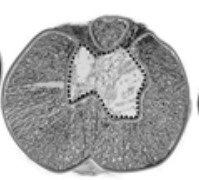

$+1.5$
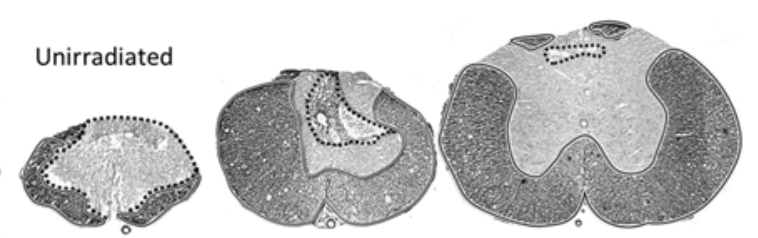

X-irradiated

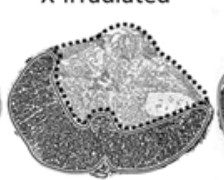

0

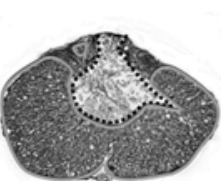

$-1.5$

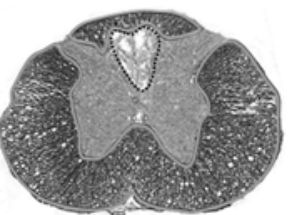

$-3.5$

B
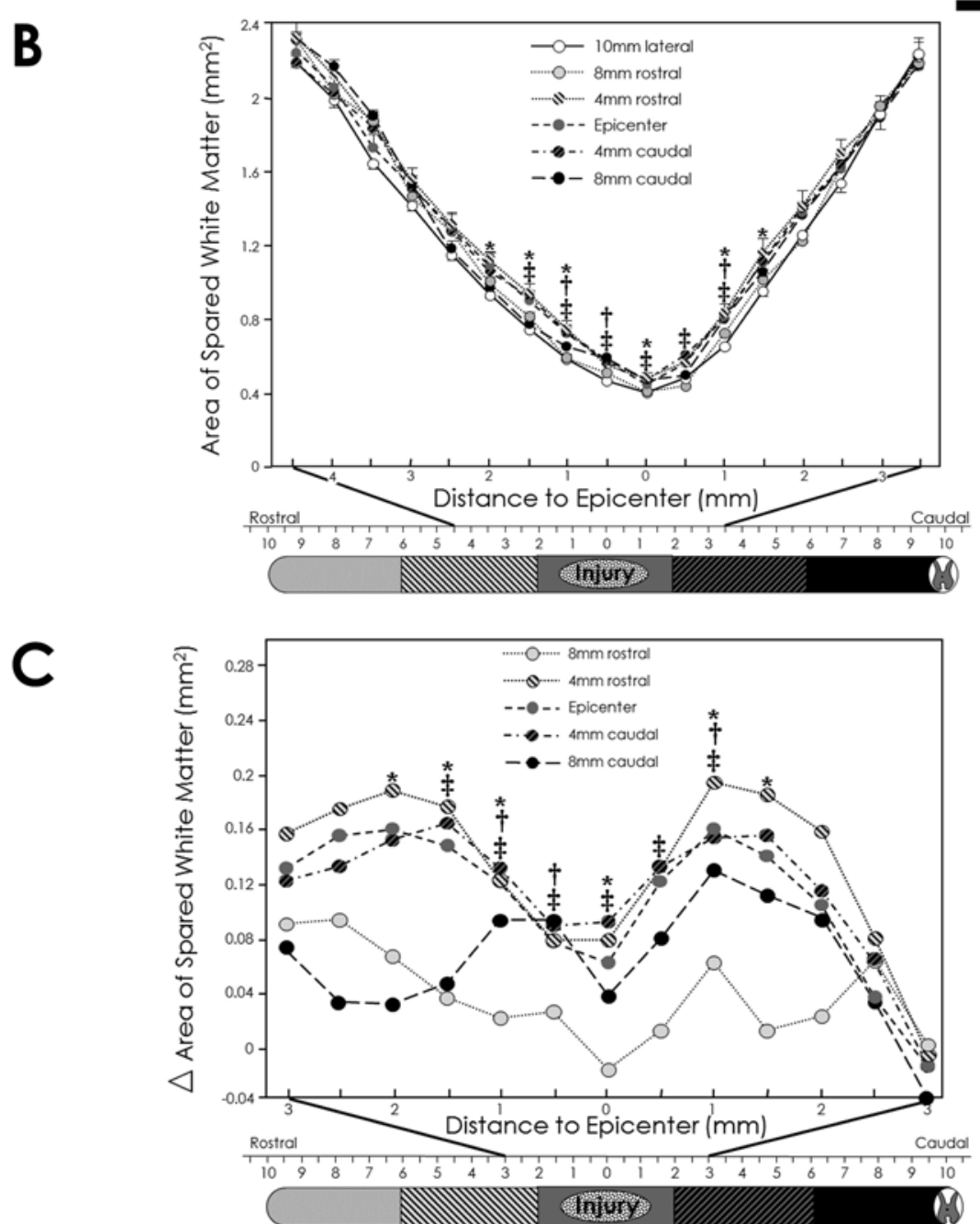

FIG. 2. A: Transverse sections of spinal cords at the contusion epicenter and 1.5 or $3.5 \mathrm{~mm}$ rostral (+) or caudal (-) to the epicenter (0) from an unirradiated rat (upper micrographs) and a rat x-irradiated at $4 \mathrm{~mm}$ rostral to the epicenter (lower micrographs) at 6 weeks following contusion injury as indicated. The sections were stained with Luxol fast blue for myelin and counterstained with cresyl violet, as described in the Methods section. Typically, the central cavity and peripheral rim of spared tissue appeared histologically similar in x-irradiated and untreated spinal cords, although a greater extent of sparing of spinal cord white matter is apparent at and adjacent to the epicenter following contusion injury of the x-irradiated compared to the unirradiated spinal cord. Areas of white matter and cavitation are indicated, respectively, by striped white and dotted black lines with gray matter area remaining The calibration bar equals $1 \mathrm{~mm}$. B: The effects of varying the anatomical rostral-caudal position of the x-irradiation beam relative to the contusion site on sparing of spinal cord tissue. Values are means of determinations of the cross-sectional area of spared spinal cord white matter (in myelin-stained transverse sections) as a function of distance from the contusion epicenter. The rats received a contusion injury and radiation treatment, as described for Fig. 1A. Significant increases in spared spinal cord white matter (in a region several millimeters in length, extending rostrally and caudally from the epicenter) due to $x$-irradiation at the epicenter $(\dagger)$ and $4 \mathrm{~mm}$, but not $8 \mathrm{~mm}$, rostral $\left.{ }^{*}\right)$ and $4 \mathrm{~mm}$ caudal $(\ddagger)$ to the contusion epicenter were observed at 6 weeks postirradiation compared to rats receiving $x$-irradiation of the thorax $(p<0.05$ [least significant difference]).

FIG. 2. (continued) $\rightarrow$ 
FIG. 2. C: The effects of varying the anatomical rostral-caudal position of the $x$-irradiation beam relative to the contusion site on sparing of spinal cord tissue compared to rats receiving $x$-irradiation of the thorax. Values are means of determinations of the cross-sectional area of spared spinal cord white matter (in myelin-stained transverse sections) expressed as the difference between the x-irradiated and unirradiated spinal cord as a function of distance from the contusion epicenter. The data were smoothed with a 2-point moving average of the mean of each value and the adjacent caudal measurement. The rats received contusion injury and radiation treatment, as described for Fig. 1A. Significant increases in sparing of spinal cord white matter are seen, as in Fig. 2B.

Of note, the location of significant tissue sparing along the spinal cord was not restricted to the site of $\mathrm{x}$-irradiation but also occurred either rostrally or caudally beyond these sites into contiguous unirradiated regions of the spinal cord (Fig. 2B and C). For x-irradiation centered at 4 $\mathrm{mm}$ rostral to the contusion epicenter, significant sparing of white matter extended from $2 \mathrm{~mm}$ rostral to $1.5 \mathrm{~mm}$ caudal to the epicenter. In the case of x-irradiation centered at $4 \mathrm{~mm}$ caudal to the epicenter, significant sparing was observed in a smaller region extending from $1.5 \mathrm{~mm}$ rostral to $1 \mathrm{~mm}$ caudal to the epicenter. For x-irradiation centered at the epicenter-only segments that directly received x-irradiation-i.e., at either 0.5 or $1 \mathrm{~mm}$ rostral or $1 \mathrm{~mm}$ caudal from the epicenter-significant tissue sparing was exhibited. In general, tissue sparing occurred in a region centered near the epicenter in which tissue loss was greatest, regardless of whether x-irradiation was administered rostrally, caudally, or at the epicenter.

$\mathrm{X}$-irradiation at different levels along the spinal cord resulted in unequal sparing of spinal cord white matter containing axonal tracts, which may be differentially incorporated into circuitry-mediating locomotor recovery following injury. To determine the region where the quantitative relationship between sparing of white matter and locomotor recovery was greatest, we performed regression analysis of increases in spared spinal cord tissue against increases in final BBB scores due to $\mathrm{x}$-irradiation at $0.5-\mathrm{mm}$ intervals along the spinal cords. Significant linear regression between these variables was found at $3 \mathrm{~mm}$ (Fig. 3) $\left(\mathrm{R}^{2}=0.966\right.$, slope $=44.9 \pm 4.8 \Delta \mathrm{BBB}$ score $/ \Delta \mathrm{mm}^{2}$ spared white matter; $p=0.003), 2.5 \mathrm{~mm}\left(\mathrm{R}^{2}=0.905\right.$, slope $=26.2$ $\pm 4.9 \Delta \mathrm{BBB}$ score $/ \Delta \mathrm{mm}^{2}$ spared white matter; $\left.\mathrm{p}=0.013\right)$, and $2 \mathrm{~mm}\left(\mathrm{R}^{2}=0.888\right.$, slope $=21.7 \pm 4.5 \Delta \mathrm{BBB}$ score $/ \Delta$ $\mathrm{mm}^{2}$ spared white matter; $\mathrm{p}=0.017$ ) rostral to the injury epicenter. In addition, the coefficient of determination, a measure of the degree to which tissue sparing is predictive of locomotor score, was maximal at $3 \mathrm{~mm}$ rostral from the epicenter $\left(R^{2}=0.966\right)$. The coefficient of determination decreased with measurements at intervals along the spinal cord in the caudal direction, with the lowest values found near the epicenter, consistent with the greatest correlation between white matter sparing and locomotor recovery in a region $2-3 \mathrm{~mm}$ rostral from the epicenter.

\section{Discussion}

This study demonstrates that external-beam x-irradiation of spinal cord segments either at or directly caudal or rostral to the contusion epicenter produces significant recovery of locomotor function. The response to irradiating
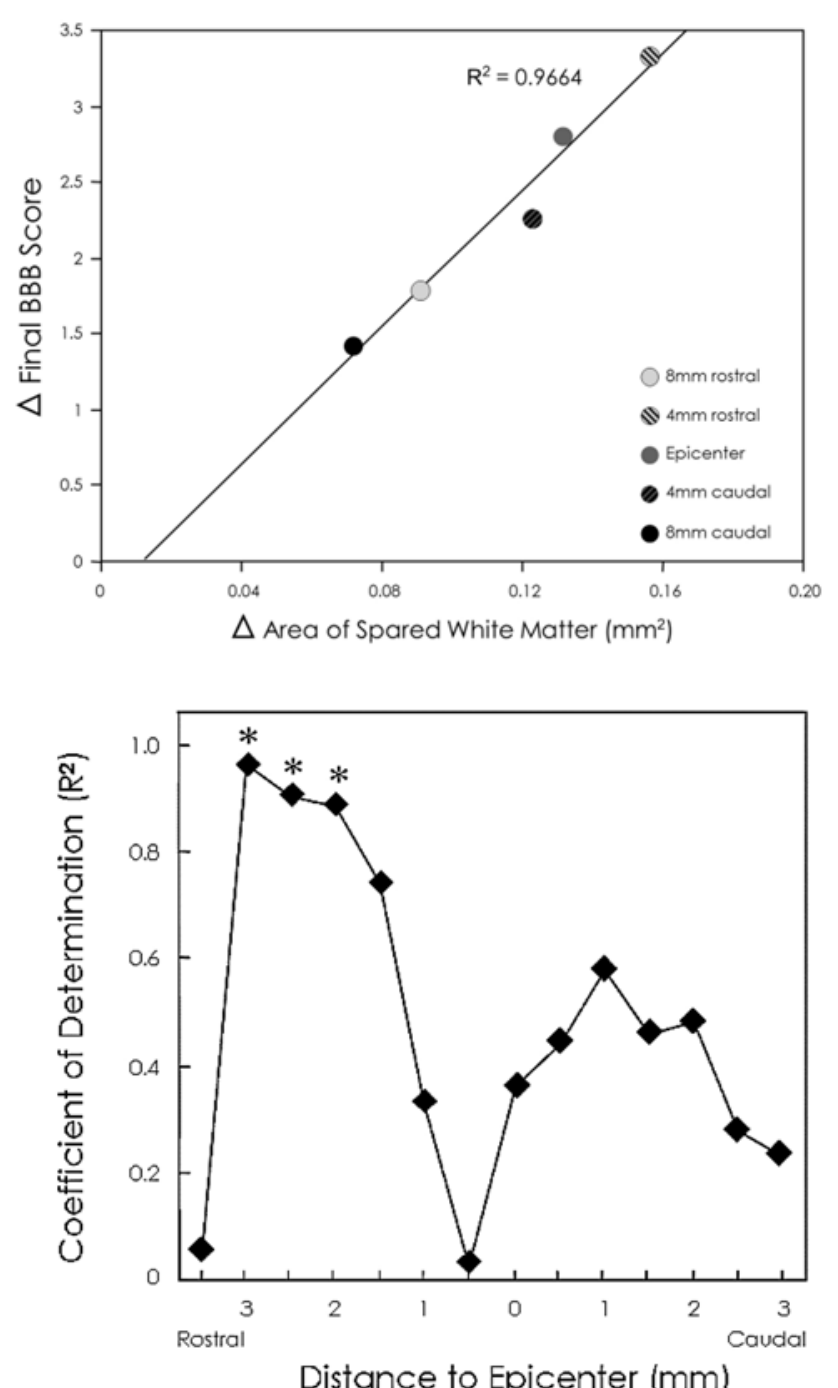

FIG. 3. Upper: A regression analysis of increases in spared spinal cord tissue at $3 \mathrm{~mm}$ rostral to the contusion epicenter against increases in final BBB scores due to $x$-irradiation of segments at the epicenter or 4 or $8 \mathrm{~mm}$ rostral or caudal to the epicenter was performed as indicated. Significant linear regression between these variables was found $\left(R^{2}\right.$ $=0.966$, slope $=44.9 \pm 4.8 \Delta \mathrm{BBB}$ score $/ \Delta \mathrm{mm}^{2}$ spared white matter; $p=0.003)$. Lower: The coefficient of determination $\left(R^{2}\right)$ was plotted as a function of distance from the contusion epicenter. The coefficient of determination decreased with measurements at intervals along the spinal cord in the caudal direction with the lowest values found near the epicenter, consistent with the greatest correlation between white matter sparing and locomotor recovery in a region $2-3 \mathrm{~mm}$ rostral from the epicenter where significant regression was found $\left({ }^{*} p<0.05\right.$, as in Fig. 3 upper).

a 4-mm length of spinal cord was similar to irradiating a much longer 15-mm length of injured spinal cord centered at the contusion epicenter. ${ }^{32}$ The present results indicate that the minimum therapeutic target does not necessarily include the contusion epicenter, since irradiation of adjacent spinal cord segments either rostrally or caudally produced significant increases in locomotor recovery. Furthermore, the spinal cord must be irradiated at least at some location in order to enhance locomotor recovery, since irradiation of the thorax, rather than the spinal cord, 
led to inferior recovery similar to unirradiated rats. The finding of this minimal target has potential therapeutic significance, since it allows a greatly reduced target volume so that uninjured tissue is not needlessly irradiated.

In the present study, as well as several previous studies, ${ }^{14,30}$ injured spinal cords were irradiated effectively with a dose of $20 \mathrm{~Gy}$. However, in our study of stereotactic $\mathrm{x}$-irradiation, ${ }^{32}$ a lower dose of $10 \mathrm{~Gy}$ was effective, whereas a dose of $20 \mathrm{~Gy}$ was not. This apparent discrepancy may be explained by a large difference in the dose rates used in these studies. The 20-Gy doses were delivered at rates of 1.5-2.5 Gy/min, whereas the 10-Gy dose was delivered at a much faster rate of $6.4 \mathrm{~Gy} / \mathrm{min}$, which, in turn, is related to the respective use of lower $(90-320 \mathrm{kV})$ and higher $(6 \mathrm{MeV})$ energies of radiation. More recently, in a study ${ }^{25}$ involving irradiation of the vestibular nerve with a dose of approximately $17 \mathrm{~Gy}$, the dose rate was found to be an important variable in irradiating nervous tissue since deleterious effects-i.e., facial nerve dysfunction and hearing loss-were found to be greater when irradiating at dose rates higher, but not lower, than $2.7 \mathrm{~Gy} / \mathrm{min}$. This radiation-induced toxicity to nervous tissue found at higher dose rates would be consistent with a dose of $20 \mathrm{~Gy}$ being effective for treating SCI when delivered at a lower, but not a higher, dose rate, as has been observed.

A consistent feature of studies using this experimental model of spinal cord contusion injury is that the magnitude of locomotor recovery is closely paralleled by the extent of tissue sparing measured at the contusion epicenter. ${ }^{2,3,21,28-31}$ However, in the present experiments, although there was significant sparing at the epicenter, greater sparing was observed rostral and caudal to the epicenter. In addition, the correlation between the degree of white matter sparing and the extent of locomotor recovery following $\mathrm{x}-$ irradiation was greatest in a region rostral to the epicenter, reduced caudally and minimal at the epicenter. This relationship provides evidence that, although sparing occurred both rostrally and caudally with respect to the epicenter, sparing of axonal tracts rostral to the injury is most important for locomotor recovery.

Why might sparing of white matter immediately rostral to the injury site be more directly related to the degree of locomotor recovery? It is possible that this is due to sparing of axons of long descending pathways, including those of corticospinal and propriospinal neurons that incorporate into detour or relay circuits believed to be necessary for functional recovery following SCI ${ }^{1,7}$ Previous studies have shown the importance of the formation of propriospinal relay connections involving propriospinal neurons located rostral to the injury site for locomotor recovery, since ablation of these neurons, although without effect in uninjured rats, prevented locomotor recovery following injury. The connectivity of these rostral propriospinal neurons, as shown by retrograde labeling, is also increased following contusion injury in mice in which locomotor recovery is improved by transgenic inactivation of astroglial NF- $\mathrm{\kappa B}$. ${ }^{6}$ In addition, there is evidence that corticospinal axons exhibit increased sprouting, entry into the gray matter, and synaptogenesis immediately rostral to the injury site in a model of injury in which motor recovery is enhanced by chondroitinase treatment combined with rehabilitation. ${ }^{26}$
Together, this evidence suggests that this rostral region contains key circuitry essential for locomotor recovery and therefore represents an important therapeutic target for opposing paralysis.

The results of this study also indicate that $\mathrm{x}$-irradiation can exert neuroprotection in contiguous unirradiated areas of the spinal cord, either rostrally or caudally, relative to the $\mathrm{x}$-irradiation site. For example, $\mathrm{x}$-irradiation $4 \mathrm{~mm}$ rostral to the epicenter led to sparing of white matter caudal to the epicenter and vice versa. To explain the ability of $\mathrm{x}$ irradiation to affect unirradiated regions, it is necessary to hypothesize that signaling occurs between the irradiated and unirradiated portions of the spinal cord. Considerable experimental evidence has accumulated demonstrating that $\mathrm{x}$-irradiation exerts bystander effects beyond the site of irradiation, which can be either destructive or exert beneficial protective effects. Previously it was thought that the cell nucleus needed to be irradiated in order for the radiation to be biologically effective. However, biological effects are exerted in bystander tissue without nuclear irradiation and this has been observed in cells in which only the cytoplasm was irradiated. ${ }^{12}$ Evidence implicates a variety of factors that mediate bystander effects, including reactive oxygen species, nitric oxide, calcium, and cytokines that may be transmitted via gap junctions or by autocrine/ paracrine or juxtacrine mechanisms. Bystander effects can be exerted at considerable distances. In one experiment, lead shielding was used to unilaterally irradiate mice to demonstrate that the DNA of shielded skin could be affected by irradiation to a similar extent as unshielded skin and that this effect could travel at least a centimeter into the shielded region. ${ }^{17}$ In other experiments, irradiation of the head affected the testis. ${ }^{12}$

One possibility for communication with unirradiated regions of the spinal cord is that $\mathrm{x}$-irradiation may generate a substance or substances that can diffuse rostrally or caudally to exert neuroprotection and improve locomotor recovery. The identity of the putative diffusible metabolite could be astrocytic glutathione or the severalfold more potent nitrosoglutathione generated from nitric oxide, itself a product of x-irradiation ${ }^{18}$ and glutathione, which can diffuse between cells and are both known to be neuroprotective. ${ }^{15,16}$ The route of diffusion may include efflux from astrocytes via transporters or hemichannels, although diffusion through gap junctions between cells such as astrocytes is also possible. On arrival at distant sites, there is a "shuttle" mechanism to internalize extracellular glutathione involving generating transportable intermediates such as cysteinylglycine. ${ }^{8}$

A factor that may be related to the unequal neuroprotective effects of $\mathrm{x}$-irradiation along the spinal cord is that there are rostral-caudal gradients of tissue damage that have been described that, in turn, could be related to the differential effectiveness of localized treatment. In particular, the amount of damage appears greatest rostrally. This is shown in experiments in which the short-term effects of SCI in impairing blood flow and causing hemorrhage and ischemia have a rostral-caudal gradient that is greatest rostral to the injury site..$^{22}$ This is also shown in longerterm experiments in which the extent of tissue sparing was compared and found to be greater caudally and reduced 
rostrally.$^{13,22,27,28}$ On the other hand, in cases in which various neuroprotective treatments were administered and the extent of sparing was compared, greater sparing was observed rostrally. ${ }^{13,28}$ Together, these studies indicate greater damage and repair rostrally. The minimal effectiveness of $\mathrm{X}$-rays at the epicenter may simply reflect the greater damage to tissue that leads to decreased salvageable tissue. At distances of $8 \mathrm{~mm}$ rostral or caudal, sparing is greatly reduced, since this is probably uninjured so that there is much less tissue to spare.

An important safety issue regarding $\mathrm{x}$-irradiation of the spinal cord is the possibility of long-term deleterious effects of ionizing radiation. Of particular concern is the potential for later development of paralysis due to myelopathy that may result from eventual depletion of oligodendrocyte precursor cells in the irradiated regions of the spinal cord. However, the occurrence of this demyelination-induced paralysis is determined by the "dose-volume effect" in which the threshold dose of radiation required to induce tissue damage is inversely related to the volume of tissue irradiated. ${ }^{5}$ Stereotactic radiosurgery was originally developed to take advantage of this relationship so that by targeting limited volumes of tissue the tolerance for higher doses of radiation is increased. In a study demonstrating the dose-volume effect in the spinal cord of the rat, lengths of cord of 2, 4, 8, or $20 \mathrm{~mm}$ were exposed to varying doses of radiation of up to 112 Gy to determine the $\mathrm{ED}_{50}(50 \%$ effective dose) for inducing paralysis within a 210-day period. ${ }^{5}$ The $\mathrm{ED}_{50}$ for lengths of 2 or $4 \mathrm{~mm}$ (87.8 and 53.7 Gy, respectively) was greatly increased compared to cord lengths of 8 or $20 \mathrm{~mm}$ (24.9 and $20.4 \mathrm{~Gy}$, respectively) and did not overlap, demonstrating that small volumes of spinal cord are highly resistant to the long-term effects of irradiation. Similarly, others have found in mice that irradiation of a relatively long cord length of $22 \mathrm{~mm}$ also results in myelopathy with comparable doses of approximately $20 \mathrm{~Gy}$ within 360 days. ${ }^{19}$ Although the target cells involved are not definitively known, it is suspected that oligodendrocyte precursor cells or endothelial cells are adversely affected by irradiation, causing myelopathy. It has been found that precursors originating from surrounding white matter can migrate $2 \mathrm{~mm}$ into a demyelinated region, allowing for remyelination. ${ }^{9}$ In the present studies, the irradiated region had a diameter of $4 \mathrm{~mm}$, which would allow for remyelination of any demyelination that might occur; however, the therapeutic dose of $20 \mathrm{~Gy}$ is much lower than that required to produce myelopathy within this tissue volume. Consistent with this, safety studies of more than 1000 patients treated with stereotactic radiosurgery for spinal cord tumors have found that myelopathic complications are rare and only occurred in $0.6 \%$ of the patients. ${ }^{10}$ However, this small percentage of patients may have been prone to myelopathy by factors such as prior $\mathrm{x}$-irradiation, $\mathrm{x}$-irradiation of large cord volumes, chemotherapy, antiangiogenic treatment, or genetic predisposition. Thus, targeted x-irradiation of small volumes of spinal cord tissue for the purpose of therapy for SCI should be particularly safe.

The possibility of malignancy due to x-irradiation of the spinal cord is also a safety concern. However, a study of rats x-irradiated with 15-32 Gy in the cervical region of the spinal cord found no spinal cord tumors at 640 days, although in surrounding tissue there was an increased incidence of rhabdomyosarcoma and other sarcomas. ${ }^{24}$ On the other hand, since stereotactic radiosurgery can specifically target the spinal cord, this risk of carcinogenesis in surrounding tissues can be avoided. Another concern is the possibility that astrocytic gliosis may result from potential injury due to irradiation. However, studies of experimental SCI have shown that the dose of $\mathrm{x}$-irradiation used in the present studies, i.e., $20 \mathrm{~Gy},{ }^{14}$ or a higher dose of $40 \mathrm{~Gy},{ }^{33}$ largely prevents astrogliosis as well as fibrotic scarring from occurring in the injured spinal cord.

\section{Conclusions}

These results indicate that $\mathrm{x}$-irradiation in a region rostral to the injury epicenter is optimal for recovery from SCI. This may be due to sparing of axons of corticospinal or propriospinal neurons that incorporate into circuits necessary for functional recovery following SCI. The novel finding of this minimal target has potential therapeutic significance since it allows for a greatly reduced target volume so that uninjured tissue is not needlessly irradiated. Optimization of the use of stereotactic x-irradiation either alone or in combination with additional agents may further increase recovery from contusion injury.

\section{Acknowledgments}

This work was supported by grants (to Richard J. Zeman) from the National Institutes of Health (R43 NS047760) and the United States Department of Defense (W81XWH-05-1-0076).

\section{References}

1. Bareyre FM, Kerschensteiner M, Raineteau O, Mettenleiter TC, Weinmann O, Schwab ME: The injured spinal cord spontaneously forms a new intraspinal circuit in adult rats. Nat Neurosci 7:269-277, 2004

2. Basso DM, Beattie MS, Bresnahan JC: Graded histological and locomotor outcomes after spinal cord contusion using the NYU weight-drop device versus transection. Exp Neurol 139:244-256, 1996

3. Basso DM, Beattie MS, Bresnahan JC: A sensitive and reliable locomotor rating scale for open field testing in rats. J Neurotrauma 12:1-21, 1995

4. Basso DM, Beattie MS, Bresnahan JC, Anderson DK, Faden AI, Gruner JA, et al: MASCIS evaluation of open field locomotor scores: effects of experience and teamwork on reliability. Multicenter Animal Spinal Cord Injury Study. J Neurotrauma 13:343-359, 1996

5. Bijl HP, van Luijk P, Coppes RP, Schippers JM, Konings AW, van der Kogel AJ: Dose-volume effects in the rat cervical spinal cord after proton irradiation. Int J Radiat Oncol Biol Phys 52:205-211, 2002

6. Brambilla R, Hurtado A, Persaud T, Esham K, Pearse DD, Oudega M, et al: Transgenic inhibition of astroglial NFkappa B leads to increased axonal sparing and sprouting following spinal cord injury. J Neurochem 110:765-778, 2009

7. Courtine G, Song B, Roy RR, Zhong H, Herrmann JE, Ao Y, et al: Recovery of supraspinal control of stepping via indirect propriospinal relay connections after spinal cord injury. Nat Med 14:69-74, 2008

8. Dringen R, Pfeiffer B, Hamprecht B: Synthesis of the antioxidant glutathione in neurons: supply by astrocytes of CysGly as precursor for neuronal glutathione. J Neurosci 19:562569,1999 
9. Franklin RJ, Gilson JM, Blakemore WF: Local recruitment of remyelinating cells in the repair of demyelination in the central nervous system. J Neurosci Res 50:337-344, 1997

10. Gibbs IC, Patil C, Gerszten PC, Adler JR Jr, Burton SA: Delayed radiation-induced myelopathy after spinal radiosurgery. Neurosurgery 64 (2 Suppl):A67-A72, 2009

11. Gruner JA: A monitored contusion model of spinal cord injury in the rat. J Neurotrauma 9:123-128, 1992

12. Hamada N, Maeda M, Otsuka K, Tomita M: Signaling pathways underpinning the manifestations of ionizing radiationinduced bystander effects. Curr Mol Pharmacol 4:79-95, 2011

13. Hillard VH, Peng H, Zhang Y, Das K, Murali R, Etlinger JD, et al: Tempol, a nitroxide antioxidant, improves locomotor and histological outcomes after spinal cord contusion in rats. J Neurotrauma 21:1405-1414, 2004

14. Kalderon N, Fuks Z: Structural recovery in lesioned adult mammalian spinal cord by $\mathrm{x}$-irradiation of the lesion site. Proc Natl Acad Sci U S A 93:11179-11184, 1996

15. Kamencic H, Griebel RW, Lyon AW, Paterson PG, Juurlink $\mathrm{BH}$ : Promoting glutathione synthesis after spinal cord trauma decreases secondary damage and promotes retention of function. FASEB J 15:243-250, 2001

16. Khan M, Sekhon B, Giri S, Jatana M, Gilg AG, Ayasolla K, et al: S-Nitrosoglutathione reduces inflammation and protects brain against focal cerebral ischemia in a rat model of experimental stroke. J Cereb Blood Flow Metab 25:177-192, 2005

17. Koturbash I, Rugo RE, Hendricks CA, Loree J, Thibault B, Kutanzi K, et al: Irradiation induces DNA damage and modulates epigenetic effectors in distant bystander tissue in vivo. Oncogene 25:4267-4275, 2006

18. Leach JK, Black SM, Schmidt-Ullrich RK, Mikkelsen RB: Activation of constitutive nitric-oxide synthase activity is an early signaling event induced by ionizing radiation. J Biol Chem 277:15400-15406, 2002

19. Lo YC, McBride WH, Withers HR: The effect of single doses of radiation on mouse spinal cord. Int J Radiat Oncol Biol Phys 22:57-63, 1992

20. Narayana PA, Grill RJ, Chacko T, Vang R: Endogenous recovery of injured spinal cord: longitudinal in vivo magnetic resonance imaging. J Neurosci Res 78:749-759, 2004

21. Noble LJ, Wrathall JR: Spinal cord contusion in the rat: morphometric analyses of alterations in the spinal cord. Exp Neurol 88:135-149, 1985

22. Ohashi T, Morimoto T, Kawata K, Yamada T, Sakaki T: Correlation between spinal cord blood flow and arterial diameter following acute spinal cord injury in rats. Acta Neurochir (Wien) 138:322-329, 1996

23. Qian T, Guo X, Levi AD, Vanni S, Shebert RT, Sipski ML: High-dose methylprednisolone may cause myopathy in acute spinal cord injury patients. Spinal Cord 43:199-203, 2005

24. Sminia P, Haveman J, Jansen W, Hendriks JJ, van Dijk JD: Hyperthermia promotes the incidence of tumours following $\mathrm{X}$-irradiation of the rat cervical cord region. Int J Radiat Biol 60:833-845, 1991

25. Smith DR, Saadatmand HJ, Wu CC, Black PJ, Wuu YR, Lesser J, et al: Treatment outcomes and dose rate effects following gamma knife stereotactic radiosurgery for vestibular schwannomas. Neurosurgery 85:E1084-E1094, 2019
26. Wang D, Ichiyama RM, Zhao R, Andrews MR, Fawcett JW: Chondroitinase combined with rehabilitation promotes recovery of forelimb function in rats with chronic spinal cord injury. J Neurosci 31:9332-9344, 2011

27. Ward RE, Huang W, Kostusiak M, Pallier PN, Michael-Titus AT, Priestley JV: A characterization of white matter pathology following spinal cord compression injury in the rat. Neuroscience 260:227-239, 2014

28. Zeman RJ, Bauman WA, Wen X, Ouyang N, Etlinger JD, Cardozo CP: Improved functional recovery with oxandrolone after spinal cord injury in rats. Neuroreport 20:864-868, 2009

29. Zeman RJ, Feng Y, Peng H, Etlinger JD: Clenbuterol, a $\beta\left({ }_{2}\right)$ adrenoceptor agonist, improves locomotor and histological outcomes after spinal cord contusion in rats. Exp Neurol 159:267-273, 1999

30. Zeman RJ, Feng Y, Peng H, Visintainer PF, Moorthy CR, Couldwell WT, et al: X-irradiation of the contusion site improves locomotor and histological outcomes in spinal cordinjured rats. Exp Neurol 172:228-234, 2001

31. Zeman RJ, Peng H, Feng Y, Song H, Liu X, Etlinger JD: $\beta_{2}$-adrenoreceptor agonist-enhanced recovery of locomotor function after spinal cord injury is glutathione dependent. $\mathbf{J}$ Neurotrauma 23:170-180, 2006

32. Zeman RJ, Wen X, Ouyang N, Rocchio R, Shih L, Alfieri A, et al: Stereotactic radiosurgery improves locomotor recovery after spinal cord injury in rats. Neurosurgery 63:981-988, 2008

33. Zhang SX, Geddes JW, Owens JL, Holmberg EG: X-irradiation reduces lesion scarring at the contusion site of adult rat spinal cord. Histol Histopathol 20:519-530, 2005

\section{Disclosures}

The authors report no conflict of interest concerning the materials or methods used in this study or the findings specified in this paper.

\section{Supplemental Information \\ Previous Presentations}

Portions of this work appeared as a platform presentation at the Military Health Research Forum, Kansas City, Missouri, August 31,2009 , and at the 5th International Conference of the Novalis Circle, Munich, Germany, June 17, 2010.

\section{Author Contributions}

Conception and design: Zeman, Moorthy, Etlinger. Acquisition of data: Wen. Analysis and interpretation of data: Zeman, Etlinger. Drafting the article: Zeman. Reviewed submitted version of manuscript: all authors. Approved the final version of the manuscript on behalf of all authors: Zeman. Statistical analysis: Zeman. Study supervision: Zeman.

\section{Correspondence}

Richard J. Zeman: New York Medical College, Valhalla, NY. zeman@nymc.edu. 